\title{
GRAI Approach : A Methodology for Re-Engineering the Manufacturing Enterprise
}

\author{
G. DOUMEINGTS (GRAI / LAP - University Bordeaux 1 - F) \\ F. MARCOTTE (INTEGRAI - F) \\ H. ROJAS (GIAT / GITECH - F)
}

\begin{abstract}
$\underline{\text { Abstract }}$
For many companies, Business Process Re-engineering (BPR) is becoming a mean to attaining a competitive advantage. But BPR is too often related to specific processes while it should ensure a global performance by covering multi-processes. For that purpose, methodologies are required to perform an integrated Business Multi-Processes Reengineering.

GRAI approach has been developped for more than 10 years and gradually improved through a lot of industrial experiments, each of them followed by a research step. This approach allows to perform such an integrated Business Multi-Processes Reengineering.

In this paper we describe first the new requirements for BPR and then the GRAI approach. Finally, we present a recent application of the GRAI approach for the re-engineering of a workshop in the most important french company in the domain of defense industry.
\end{abstract}

\section{$\underline{\text { Key words }}$}

Business Process Re-engineering, Methodology, GRAI approach.

\section{- 1 - Introduction}

This paper presents a methodology to perform re-engineering in manufacturing enterprises. We will describe a recent application of the GRAI approach for the re-engineering of a workshop in the most important french company in the domain of defense industry.

The methodology used to perform this study allows to evaluate the weak points and the strength points according to the manufacturing requirements and the enterprise strategy. From this evaluation, a set of actions is defined to ensure the implementation of the designed solutions.

After a general introduction into the general area of Business Process Re-engineering (BPR), this paper presents the GRAI approach which is used to perform effective BPR. The third part is a presentation of a recent application in a french defense company.

In the conclusion, future development for the company and future improvements for the methodology will be discussed. 


\section{- 2 - Business Process Re-engineering (BPR)}

Companies are under increased presure today to improve performance while being customer oriented. The hight competition in a global international market and the decrease of product life cycles have led manufacturing enterprises to increase their capability, to react rapidly and with coherence, and to focuss on their core-business.

Business Process Re-engineering (BPR) is an approach for achieving improvements in a company's performance in a short period of time. BPR has helped these companies to understand how the different functions or processes are acting. Approaches exist for improving these business Processes, but many of these techniques are focussing on the improvement of individual processes (figure $1)$.

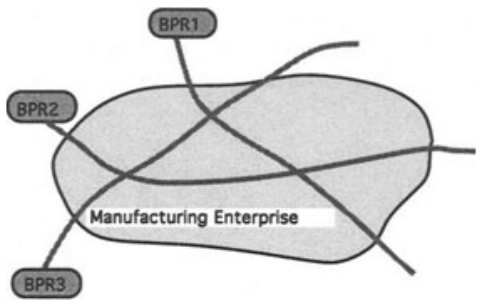

Figure 1 : Business Process Re-engineering

Now, global performance is not a set of local business performances. It is absolutly necessary to improve each business process according to the overal manufacturing enterprise performances, and not only according to the optimisation of each process individually. An efficient BPR technique must ensure Business Process Integration through a multi-process improvement (figure 2).

Company improvements are possible with manufacturing enterprise reengineering tools covering the whole re-engineering process (from evaluation to implementation) and taking into account global perfomance criteria. The GRAI approach, presented below, is an available methodology to perform such re-engineering.

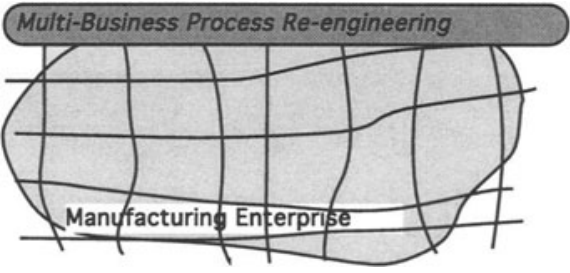

Figure 2 : Integrated Multi-Business Process Reengineering

\section{- 3 - The GRAI approach}

GRAI approach has been developped for more than 10 years and gradually improved through a lot of industrial experiments, each of them followed by a research step. Several ESPRIT projects allowed these improvements (OCS Open CAM System - ESPRIT Project $n^{\circ} 418$ ), IMPACS (Integrated Manufacturing and Planning And Control System - ESPRIT project ${ }^{\circ}$ 2338), FOF (Factory Of the Future - ESPRIT project $n^{\circ} 3143$ ), FLEXQUAR (Adaptative System for Flexible high Quality and Reliable Production - ESPRIT project $\left.{ }^{\circ} 6408\right)$. Recently, through the GLOBEMAN 21 project (Enterprise Integration for GLOBal MANufacturing towards the 21 century), an IMS (Intelligent Manufacturing System) project, an interesting study has demonstrated how GRAI approach could be used for the evaluation of manufacturing performances and for global Benchmarking.

The GRAI approach is based on the following elements 


\section{- 3 - 1 - The GRAI conceptual model of the manufacturing system}

The GRAI conceptual model aims at giving a generic description of what a manufacturing system is, on a control point of view. The control of a manufacturing system will be seen at first with a global point of view, and at the level of the decision center afterwards. The interest of GRAI conceptual model is to be generic enough in order to be usable for any kind of manufacturing system (discrete, process, and services). The GRAI conceptual model is mainly based on the system theory, control theory and hierarchical control theory.

The GRAI conceptual model identifies three systems in the manufacturing system : the physical system, the decision system and the information system. (Figure 3) The physical system has to transform raw materials, components, into the output Products through the resources.

The Decision System (DS), split up into decision making levels, according to several criteria, each level composed of one or several Decision Centers (DC). In the decisional system we determined two parts : the upper part which is periodic driven, and the lower part which is event driven. We call this second part the operating system $:$ it is the interface with the physical system : it contents the numerical control system, the programmable controllers... : this part of the DS is more event driven : we call it the operating sub-system.

The information system contains all information needed by the DS : it must be structured in an hierarchical way according to the DCs structure (Decision Centers).

\section{- 3 - 2 - The GRAI conceptual model of a Decision Center}

A decisional center at any level of the structure can be decomposed using the same criteria of decomposition: a physical, a decisional and an information (figure 4).

The controlled system is the view of the physical system at the level of decision.

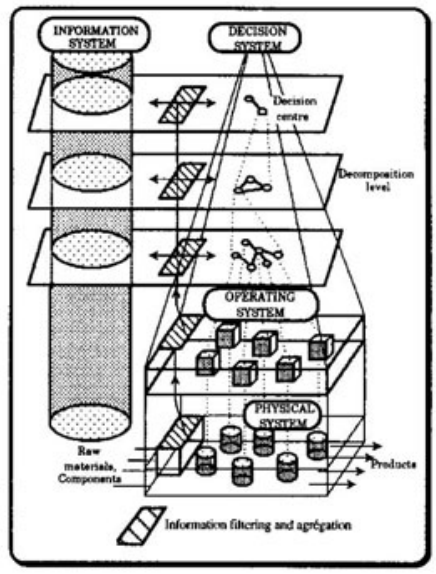

\section{Figure 3: The GRAI conceptual model of the manufacturing system}

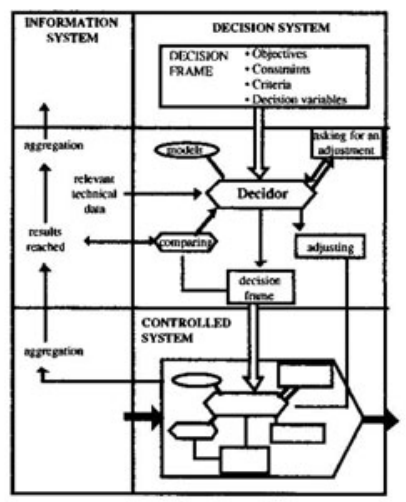

Figure 4: The GRAI

conceptual model of a Decision Center

\section{- 3 - 3 - The GRAI concept in Production Management}

The GRAI approach is focussing on the decisional aspects of the production system. It allows to model and to analyse the decisional structure of the Production Management System (PMS). In order to identify decision centres in real manufacturing system, according to the concepts defined in the GRAI conceptual model, it is necessary to use criteria, to specify the nature of the decisions and their structuration. In this study, we consider only the periodic driven part of the DS. To structure it, two axis of decomposition are defined : the vertical one is the coordination axis and the horizontal one is the synchronisation axis.

The coordination is required when considering the decomposition of the physical system. The global objective of the factory must be decomposed, step by step, in detailed objectives for the 
production cells. The temporal aspect of a production cell behaviour is different than the temporal aspect of the overall production system, and the control decisions are depending on this temporal aspect.

The criteria for the coordination is the temporal aspects of the decision : "Horizon" (The interval of time over which the decision extends (i.e. remains valid)) and "Period" (The interval of time after which we reconsider the set of decisions. In such a structure, the horizon is a sliding horizon).

The criteria for the synchronisation is a functional one. There are 3 basic types of functional activities in PMS : the product management activities (supplying decisions, stock management decisions, ...), the resource management activities (capacity management, resources allocation,...) and the planning activities. The manufacturing activity planning function defines synchronisation requirements which are objectives for the two other functions : to perform a manufacturing activity in an efficient way, we need to synchronised the availability of the products with those of the resources.

Other functions may be introduced when they are influencing the Production Management activities like : the maintenance function, the quality function, the engineering function.... Then it is possible to analyse their inter-relations which caracterize the integration of the manufacturing system.

\section{- 3 - 4 - The GRAI approach Modelling formalisms}

Based on the concepts and the criteria previously presented, the GRAI approach provides formalisms to identify the concepts in real manufacturing systems.

Three basic formalisms are used to model the manufacturing system: the GRAI grid and net, the Idefø formalism and the Entity/Relationship model.

The GRAI grid is used to model the decisionnal structure of the PMS, according to the two criteria defined previously.

The GRAI net allows to detail the decision centre.

The Idefø formalism is used to perform the functional view and to model the physical system, controlled by the PMS.

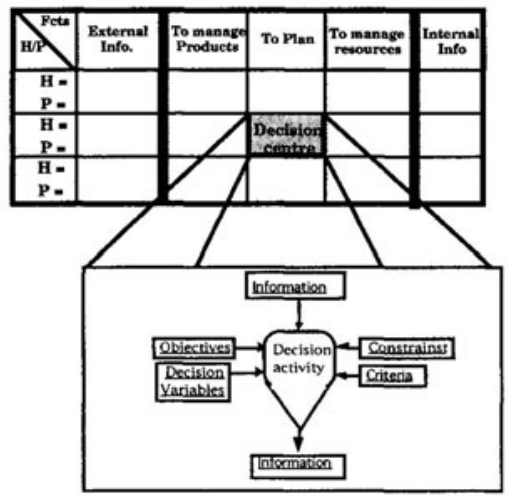

Figure 5 : The GRAI Grid and the GRAI net

Also, the E/R formalism can be used when the information system modelling is required, especially when software developments are decided.

These two last formalisms are presented in the figure 6.
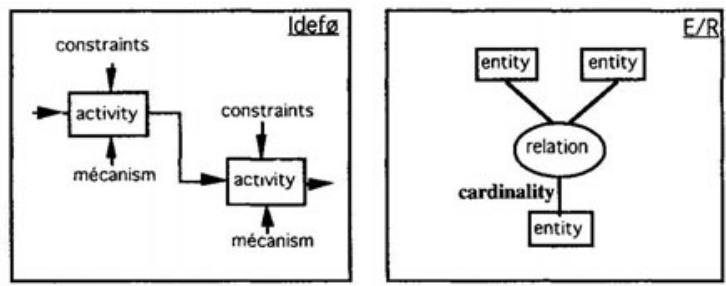

Figure $6:$ the Idefø and the E/R formalisms 


\section{- 3 - 5 - The GRAI Structured approach}

The various parts of a manufacturing enterprise must be designed in a coherent way and efficiently. For that efficiency, it is necessary to use a structured approach which provides a step by step procedure. Four main phases are identified in the GRAI approach : initialization, modelling, design and implementation. The picture 7 summarizes the overall re-engineering process.

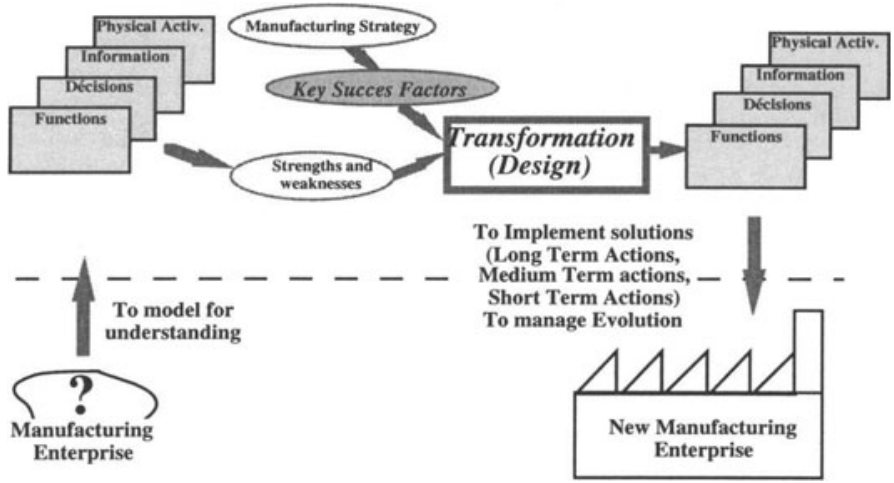

Figure 7 : Overall re-engineering process in the GRAI approach

The application of GRAI is also structured in terms of participants. It requires:

- a project board composed by the top management of the manufacturing enterprise. The goals of this group are to give the objectives of the study, and to orient the study according to the results of the main phases.

- a synthesis group composed of the main future users and main responsible people of the manufacturing system functions. This group has to follow the development of the study, and to check the results of the various stages. Their technical abilities and their suggestions will be used to guide the design of the new system.

- an analyst (several if necessary), whose job is, in particular, to collect all the data needed for the various phases.

- the users group which has to provide more detailled on specific parts of the manufacturing system.

After the modelling phase, specific groups may be defined, according to the specific solutions to be developped.

The main advantages of this approach are the following.

First, there is a set of formalisms, which allow the user to perform an integrated model of the manufacturing enterprise, according to the three views identified in the conceptual model. The GRAI grid gives an overview of the decisions made in the various functions of the manufacturing enterprise. Through the links between these decisional activities, it is possible to study the effective integration of these various functions.

Further more, for all decision centres the activities to be performed are analysed and designed according to the structure and the organisation of the physical system (the controlled system). This is to ensure the coherence between the decisional system and the physical one.

Finally, the information system is structured according to the requirements of all the decision centres. 
Second, the structured approach provides an efficient guideline to manage the project (step by step approach) and it also ensures the validity of the model which are performed (top-down analysis followed by a bottom up one).

These two main advantages give to the GRAI approach a real power to perform BPR in an integrated way.

\section{- 4 - Re-engineering of a workshop : a case study}

After the short overview of the GRAI approach, which is the basis of the study performed in a workshop of a French Defense Company, the following paragraph presents the main steps and results of the GRAI application.

\section{- 4-1-Presentation of the industrial contexte}

This workshop belongs to an important french defense company. It works in the domain of mechanical industry and in the past was acting as a subcontractor for the various divisions of the company.

Due to the tremendous changes in this industrial sector, the re-structuration of the workshop and the re-orientation of the activities of this workshop was absolutely necessary in order to find new markets. Further more, important financial losses was questionning its future, and a hard social context was bringing pressure on the top management.

The study performed with the GRAI approach was organised in three steps :

- existing situation analysis : the modelling phase,

- specification of the new workshop : the design phase,

- implementation process and planning : the action planning.

The various steps of each phase are presented below.

\section{- 4- 2 - The modelling phase}

The first phase was the modelling phase in which we have elaborated the model of the workshop using the knowledge of the actors. To perform this modelling phase two groups were working in parallel : the Synthesis Group and the Foreman Group.

The Synthesis Group was composed of the responsible persons of the main functions of the workshop : Purchasing, Engineering, Manufacturing, Supplying, Quallity and Maintenance functions ( 8 persons). This group was in charge of analysing and modelling the overall organisation of the workshop : main decision centres, relations between functions, hierarchical structure and coherence of the PMS.

Based on this global view, the Foreman Group (equivalent to the users group) was in charge of analysing in detail specific complex decision centres at the short term level, or specific manufacturing cell organisation. This group was composed of the main Foreman or team leaders, working in the workshop ( 7 persons).

The global analysis with the Synthesis Group allows to identify the points to be analysed in detail with the Foreman Group. Such an approach allows to focuss on the real problems, and it avoids to be swamped by a mass of irrelevant detail, which may cost time and money.

To support this phase, 2 persons from INTEGRAI (the research transfert company of GRAI) was guiding the sudy and managing the meetings, and 1 person from the company had been allocated to this study to learn from the GRAI approach and to perform interviews and complementary data collection. 
The objectives of the modelling phase are to determine the improvement tracks to be studied during the design phase. For that purpose, the study was organised in three steps :

- training to modelling techniques,

- application to the workshop,

- analysis of the models to evaluate the workshop situation.

The modelling phase provides a set of models :

- functional model of the workshop (Idef $\varnothing$ formalisms),

- physical model of the workshop (Idefø formalisms),

- decisional model of the management structure (GRAI grid and nets formalisms),

- information system (Entity/Relation formalisms).

From these models, strengths and weaknesses are identified. The evaluation is performed on the following aspects :

- production management structure (decisional level and functional decomposition),

- coordination principles (decision frames, information flows),

- production flow analysis (physical system organisation).

This evaluation has also been performed according to the business planning of the company. However, this business planning was not precise enough, and the information was not enough disseminated.

From this evaluation, seven tracks have been identified, as being important to improve the performance of the workshop :

- strategic orientations and commercial actions,

- structuration of the physical system,

- organisation of the "Scheduling" department,

- implementation of a hirarchical planning system,

- product quality control activity definition,

- tools to support customer order engineering,

- training on benchmarking procedures.

\section{- 4 - 3 - The design phase}

Before to start the design phase, the business planning of this workshop has been collected. This business planning was deduced from a market analysis and also from the evaluation of the strong points of the physical system (products and processes).

\section{- Design phase : organisation}

The design phase objectives was to re-inforce the training on the weak points, to look for practical solutions and to define the planning to implement the selected solutions.

The organisation of this phase has been made according to the 7 improvements tracks. For each track, a working group has been created, with participants from the both groups : the Synthesis Group, and the Foreman Group.

For each group, the following approach has been used :

- detailled analysis of the identified problems,

- analysis of existing studies and solution proposal,

- feasibility study,

- selected solution definition,

- proposal for an implementation planning.

Also, to ensure the overall coherence of the results, various meeting of the synthesis group and of the foreman group were organised. 


\section{- Design phase : results}

The results of the design phase is a global model describing the organisation of the workshop, based on the conclusions of each working group. This model specifies for the three subsystems (Physical, Informational and Decisional) the physical system organisation, the relations between the various functions of the workshop, and the responsabilities to be allocated.

\section{Physical system :}

Based on the evaluation of the current situation, and based on the business planning, a product oriented organisation has been selected. A detailled analysis has shown that the current available machine-tools were sufficient to ensure the constitution of the production cells (figure 8). A geographical implementation has been proposed, taking into account the technical constrainsts related to specific machines. One constrainst has led us to identify one common cell for the last activities of some manufacturing processes (especially quality checking activities). Training requirements and the new team organisation was also defined.

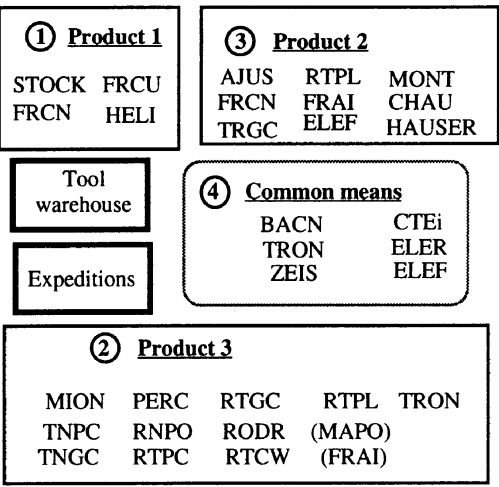

Figure 8 : Production cell constitution

\section{Decisional system:}

The interest of the GRAI approach is to allow an integrated modelling. So based on the structure of the physical system and based on the hirarchical planning principles, we have specified the decisional structure of the PMS (see figure 13). In this structure, the three basic planning levels are defined, and the relations between the various functions to.

The detailled description of each planning level is based on the physical system, as it is seen by this level. From this decisional structure, the various responsabilities in each function were defined precisely (figure 9).

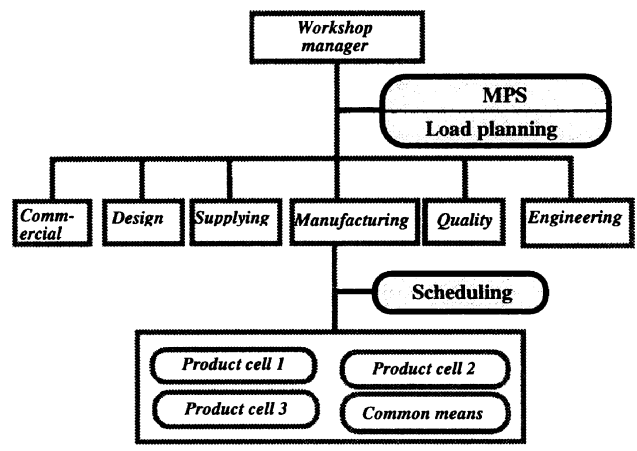

Figure 9 : Function responsabilities regarding planning levels

\section{Information system:}

In this specific case, there was no requirement for an information system anaysis. However, after the modelling phase, it appears that it was necessary to implement a scheduling system, first because of the new environment (customer order driven activity, important amount of work-orders, needs for simulation to evaluate lead-times), and also because the existing system was suppose to stop at the end of the next year.

So a specification book has been writen, three suppliers were selected, and one day has been organised with the end users to have demonstration of each selected tools.

The final choice is now on going. 


\section{- 4-4-Action planning}

The overall result of this design phase is a set of organisation principles and technical solutions. Such a result is not sufficient for a manufacturing manager, it is important to define how the solutions will be implemented.

For that purpose, we have specified a list of actions, with the related required investment, the responsible person, and the scheduling of the various steps.

These actions were defined in three groups :

- short term actions (1 month horizon)

- medium term actions ( 2 to 18 months horizon)

- long term actions (more than 18 month horizon).

\section{- 5 - Conclusion}

During this study, a BPR has been performed on the whole manufacturing enterprise. Most of the individual business processes have been studied : customer order processing, shopfloor control, quality checking, customer order engineering processes, long terme management process, product manufacturing processes,.... All these processes have been designed in a coherent way, based on the GRAI approach, which allows to relate all these processes.

Also, based on this integrated design, the manager of the workshop succeed in discussing about the CAPM to be implemented. At the beginning, the company top management was thinking about one software for all the workshops of the company, a MRP based software. Now, in this workshop, they are looking for a simple scheduling package (specification book already mentionned) which is more relevant for their type of production (specific tooling in three main areas).

Three months after the end of the application, the workshop has increased its turn over $(\approx 10 \%)$ and many improvements are in progress (level of customer service rate, number of firm orders / Number of quotations).

This study which is examplary, will support next year the application of the TIME method which is now under development in the frame of the EUREKA programme (TIME : Tools and Methods for Integration and for Management of the Evolution of Industrial Enterprises EUREKA project $\left.n^{\circ} 824\right)$. This TIME methodology aims at providing the European industrial companies with the means of supporting, guiding their long term evolution process, through modelling techniques (GIM and Game View), self audit and benchmarking. The following picture (figure 11) summarizes this conceptual elements.

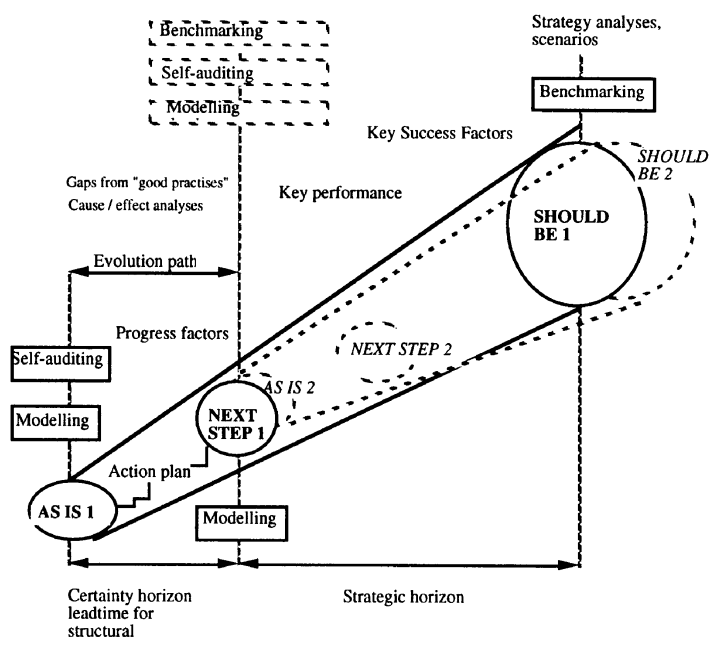

Figure 12: The TIME methodology for evolution management 


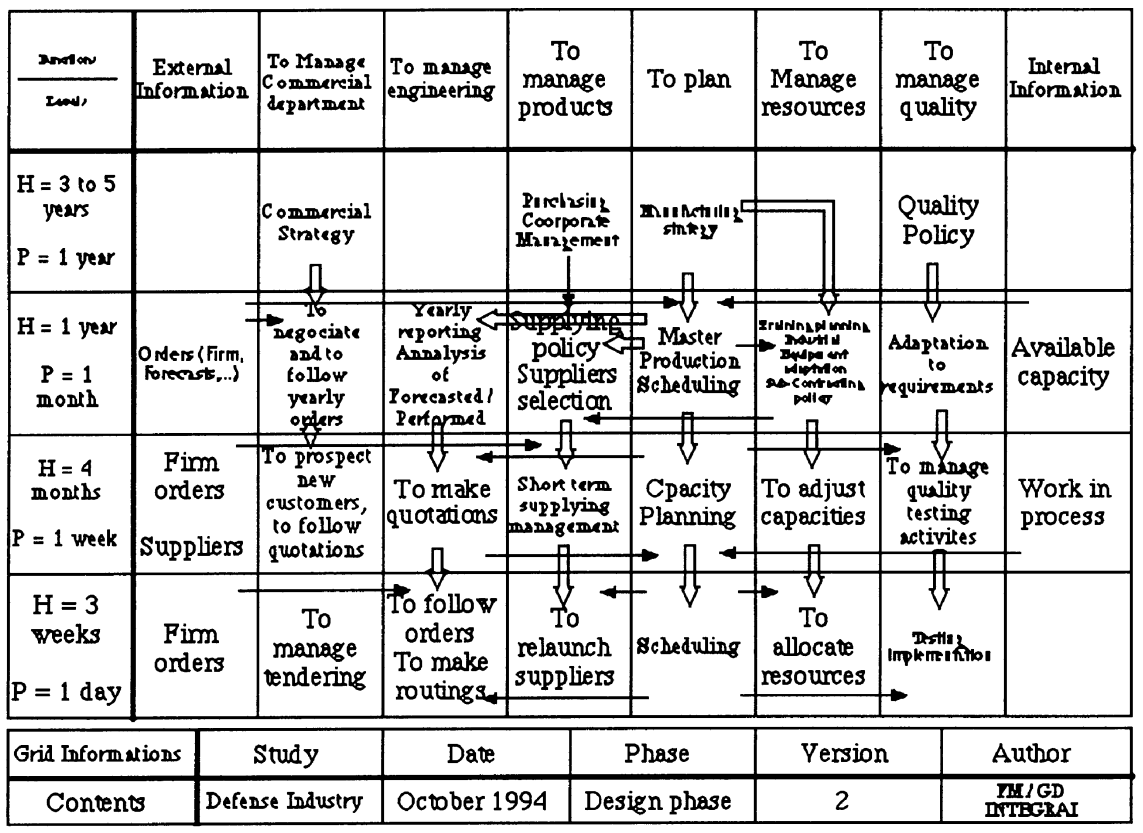

Figure 13 : the GRAI grid : the decisional structure of the PMS 